\title{
The Journal of THORACIC AND CARDIOVASCULAR SURGERY
}

\section{GENERAL THORACIC SURGERY}

\section{SIMULTANEOUSLY STAPLED LOBECTOMY: A SAFE TECHNIQUE FOR VIDEO- ASSISTED THORACIC SURGERY}

Currently, techniques for video-assisted thoracic surgery are being borrowed from the open conventional thoracotomy. However, these same techniques have made video-assisted lobectomy difficult, burdensome, and even dangerous. Simultaneously stapled lobectomy (simultaneous stapling of all hilar structures in their natural anatomic configuration) has been performed successfully in 16 patients. Every attempted simultaneously stapled lobectomy is included. The lesions included 14 malignant tumors, one giant benign pulmonary cyst, and one large necrotizing granuloma. Three right upper lobes, one right middle lobe, six right lower lobes, four left upper lobes, and two left lower lobes were resected uneventfully. Nine adenocarcinomas, two large cell carcinomas, and three squamous cell carcinomas ranging in size from 2.5 to $5 \mathrm{~cm}$ were removed. Lung fissures, the hilum, and the mediastinum were explored for lymph nodes in each patient. Median operative time was 110 minutes. Average blood loss was less than $100 \mathrm{ml}$. Median hospitalization was 6 days, although eight patients were discharged between 3 and 5 days. Three patients had air leaks for an average of 14 days and one patient had mild subcutaneous emphysema for 5 days. There was no surgical mortality. Median follow-up is 15 months (range 8 to 20 months). Simultaneously stapled lobectomy is not meant to replace conventional lobectomy by open thoracotomy. Indications are cardiac or renal problems, contralateral chest wall paralysis, neurogenic deficiencies, adamant refusal to undergo open lobectomy, psychologic aberrations, and pain from a previous thoracotomy. Contraindications include absent fissures, enlarged matted invasive nodes, fibrotic hilum, central or bulky lesions, calcific bronchi, chest wall invasion, and lesions crossing a fissure. Precedent for this technique will be

Ralph J. Lewis, MD, New Brunswick, N.J.
From the Department of Surgery, University of Medicine and Dentistry of New Jersey, Robert Wood Johnson Medical School, and the Department of Thoracic Surgery, St. Peter's Medical Center, Robert Wood Johnson University Hospital, New Brunswick, N.J.

Address for reprints: Ralph J. Lewis, MD, 185 Livingston Ave., New Brunswick, NJ 08901.
Read at the Seventy-fourth Annual Meeting of The American Association for Thoracic Surgery, New York, N.Y., April 24-27, 1994.

J ThoraC CARdiovasc SurG 1995;109:619-25

Copyright (C) 1995 by Mosby-Year Book, Inc.

$0022-5223 / 95 \$ 3.00+0 \quad \mathbf{1 2 / 6 / 6 1 6 9 6}$ 
discussed. When used with discretion in carefully selected patients, in whom an open lobectomy would be contraindicated, simultaneously stapled lobectomy might eventually prove to be another available option. Time and further experience will be necessary to determine its true merits. (J Thorac Cardiovasc Surg 1995;109: 619-25)

$A^{\mathrm{n}}$ multitude of thoracic surgical procedures have been performed successfully by means of videoassisted thoracic surgery (VATS). ${ }^{1-3}$ As the degree of complexity of these procedures increases, newer techniques more suitable for VATS must be developed and refined to ensure continued future progress in this area of thoracic surgery. ${ }^{4}$ Simultaneously stapled lobectomy has been effective for prudently selected patients needing a radical pulmonary resection.

\section{Patients and methods}

Six men and 10 women whose ages ranged from 40 to 77 years were subjected to simultaneously stapled lobectomy by means of VATS. Fourteen patients had neoplastic disease consisting of three squamous cell carcinomas, two large cell carcinomas, and nine adenocarcinomas. Two patients had benign disease; one had a large necrotizing granuloma and the other a large pulmonary cyst. Each patient had a bronchoscopic examination yielding visually and cytologically normal findings and a mediastinoscopic study revealing only benign nodes. All stations were carefully evaluated for lymph nodes in each patient. Every lymph node that was excised or sampled for biopsy was microscopically negative for malignancy. Three right upper lobectomies, one right middle lobectomy, six right lower lobectomies, four left upper lobectomies, and two left lower lobectomies were performed (Fig. 1). In each patient blood loss was less than $100 \mathrm{ml}$. Median operating time was 110 minutes (range 65 to 195 minutes). Three patients had prolonged air leaks lasting for an average of 14 days (range 10 to 17 days) and one patient had mild subcutaneous emphysema lasting 5 days. No deaths occurred. Hospitalization ranged from 3 to 21 days with eight patients being discharged between 3 and 5 days. As experience has increased, hospitalization and operating times have decreased.

Indications for simultaneously stapled lobectomy included cardiac insufficiency, renal failure necessitating dialysis, neurologic deficits with contralateral paralysis of the chest wall, severe psychologic aberrations, and rejection of surgical therapy because of persistent pain from a previous thoracotomy. Every patient made a complete recovery. One patient died 6 months after the operation of an intracranial hemorrhage attributed to hypertension. Follow-up ranges from 8 to 20 months in the 15 surviving patients. There has been no recurrence of tumor, and no patients have complained of chronic chest wall pain. Most patients returned to preoperative levels of activity within 8 to 10 days.

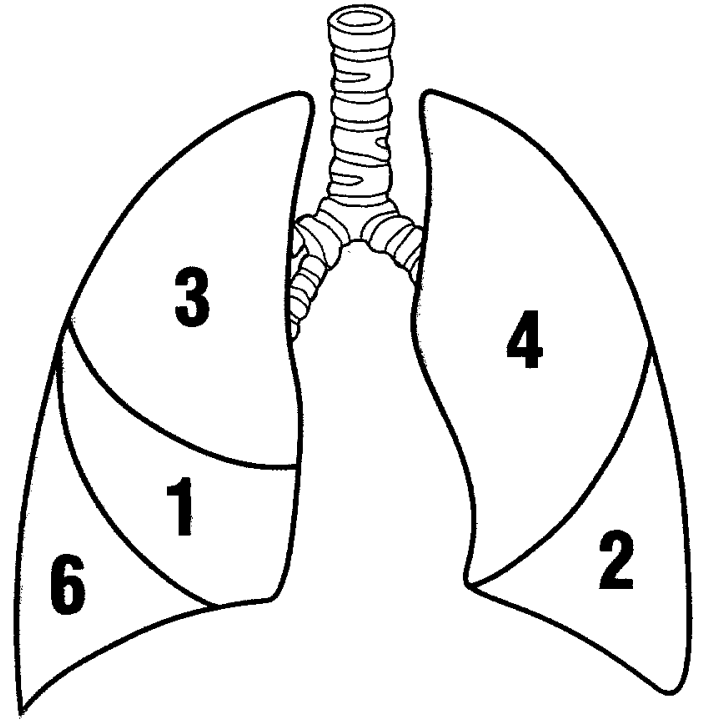

Fig. 1. Simultaneously stapled lobectomies performed.

Technique. With the patient in the lateral thoracotomy position and receiving one-lung anesthesia, a $2 \mathrm{~cm}$ incision is made in the sixth or seventh intercostal space in the midaxillary line to accommodate the scope and camera. A second incision, $2 \mathrm{~cm}$ long, is made in the posterior axillary line in the fourth intercostal space for small instruments. A third incision, 4 to $5 \mathrm{~cm}$ long, is made in the third or fourth intercostal space in the anterior axillary line for digital palpation and larger instruments (Fig. 2).

After a complete intrathoracic exploration, which includes the lung, visceral and parietal pleurae, mediastinum, and inferior pulmonary ligament, the hilar area is skeletonized and all nodal stations are carefully explored. Fissures are completed by means of blunt dissection, cautery, the Argon Beam Coagulator (Birtcher Medical Systems, Irvine, Calif.), or ELC 35 staplers (Ethicon Endosurgery, Cincinnati, Ohio). The bronchus and vessels at the base of the designated lobe are partially exposed and identified (Fig. 3). Lymph nodes when present are excised. After the appropriate lobe has been elevated with ring clamps, a No. 18 red rubber catheter is passed around the hilar structures. It is then attached to the anvil of a TL60 stapler (Ethicon Endosurgery) (Fig. 4). This will assist in guiding the stapler into proper position. Once the stapler is seated satisfactorily, the rubber catheter is removed. The stapler pin is locked into position so that all hilar structures are secured in their natural anatomic 


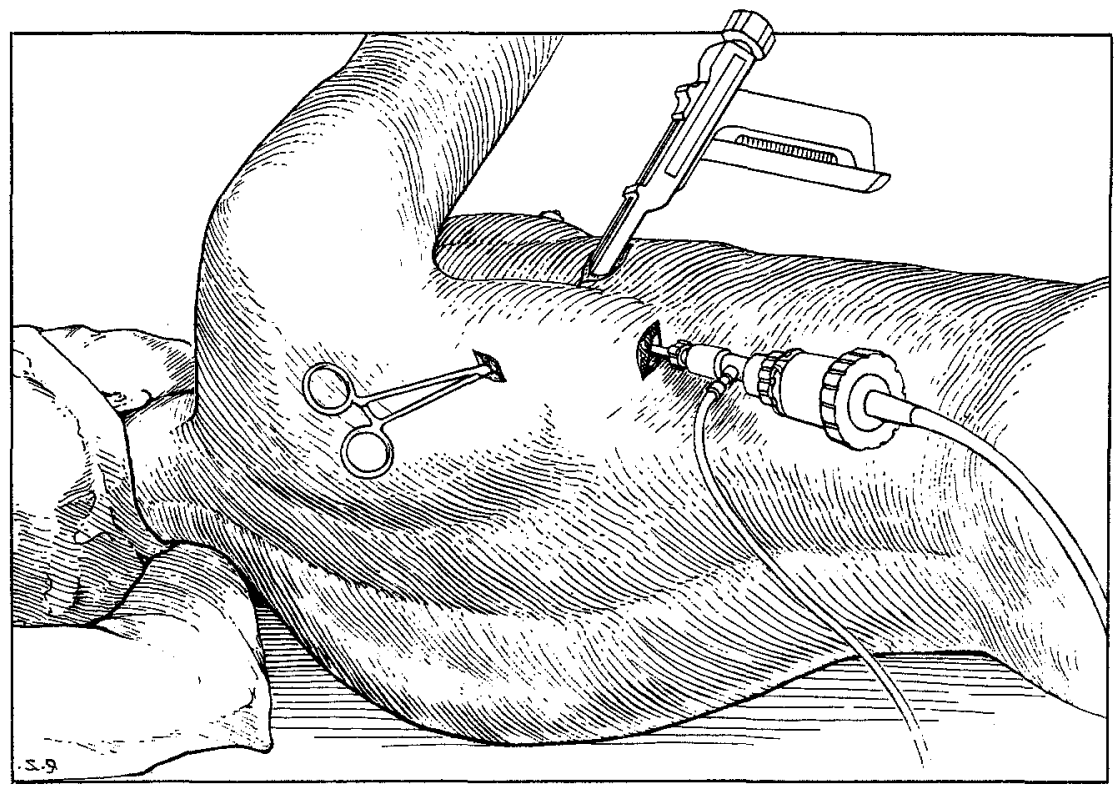

Fig. 2. Patient position and incisions.

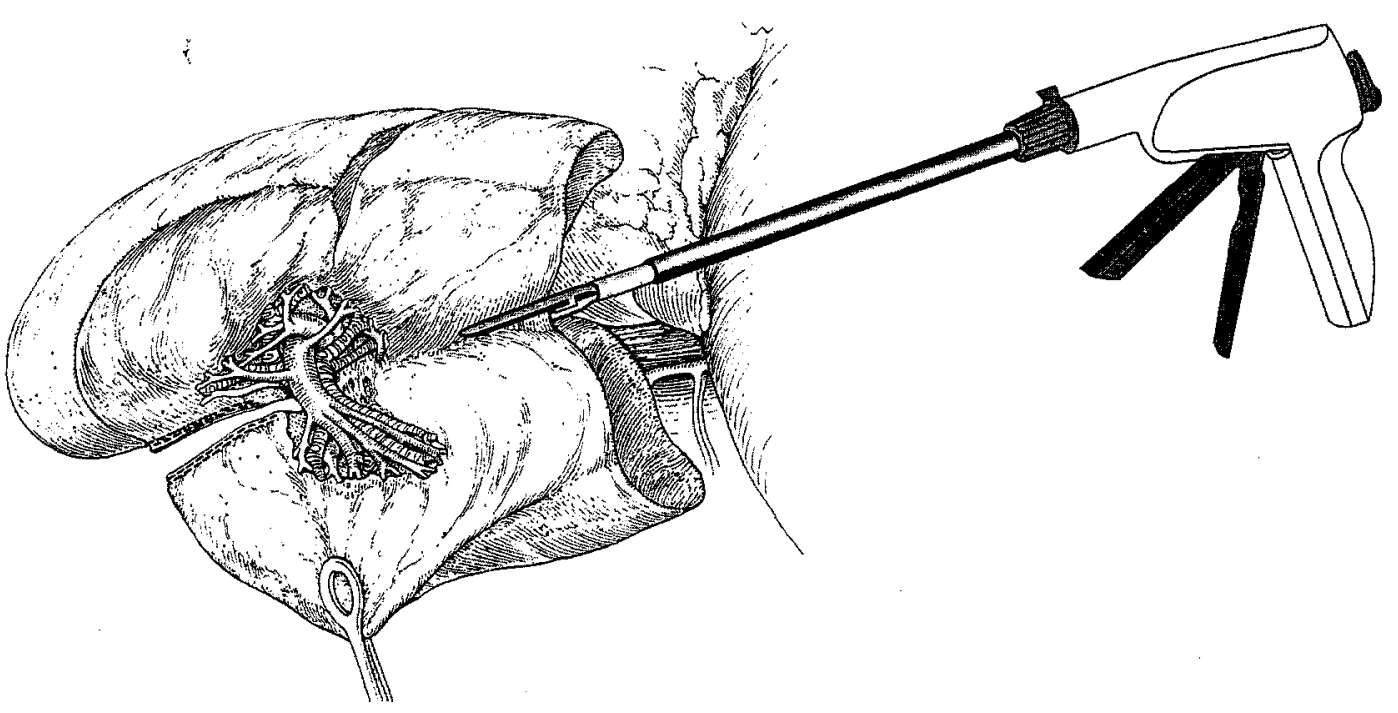

Fig. 3. Fissure completed with ELC 35 stapler. Structures at base of lobe exposed.

configuration, and the stapler is positioned proximally, close to the takeoff of the lobar bronchus. It is fired to a staple closure of $2 \mathrm{~mm}$ and then opened, removed, and reloaded. The staple line is carefully identified and its integrity confirmed. The reloaded stapler is reinserted, and a similar technique is used to place a second staple line slightly distal ( 2 to $3 \mathrm{~mm}$ ) to the first staple line.

The stapler is fired, and the lobe is excised from the closed stapler. The stapler is next slowly opened. If bleeding should occur, the stapler can be closed and appropriate measures electively pursued to maintain hemostasis. The four staple lines are carefully examined
(Fig. 5). The lobe, which has been stored away from the staple line, is then placed in a plastic bag and carefully removed through the anterior $5 \mathrm{~cm}$ incision, where the intercostal space is normally the widest, without spreading the ribs. The thorax is irrigated with saline, as the lung is partially expanded, to confirm the adequacy of the bronchial stump closure. A multiholed No. 28 red rubber catheter is tunneled through the $2 \mathrm{~cm}$ posterior incision and the midaxillary incision previously used for the scope. Pliable rubber tubes seem to cause less postoperative chest wall pain. The anterior incision is carefully closed in layers with 2-0 Vicryl suture (Ethicon, Inc., Somerville, N.J.) to the muscles, 3-0 Vicryl suture to the subcutaneous 


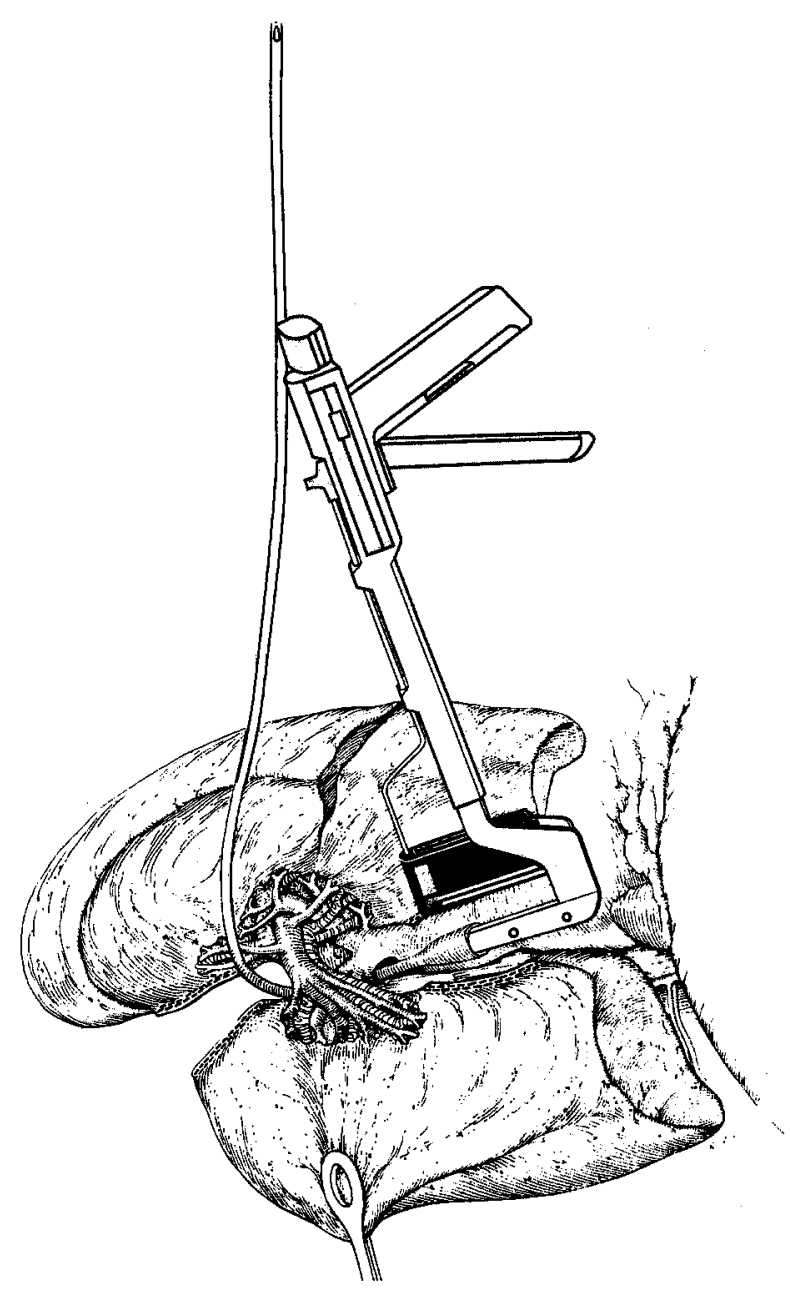

Fig. 4. TL60 stapler being guided by a red rubber catheter.

tissue, and 4-0 Vicryl suture to the subcuticular layer. Steri-Strip bandages (3M Health Care, St. Paul, Minn.) are applied. A dry sterile dressing is then placed over the small wounds.

\section{Discussion}

Simultaneously stapled lobectomy is not a modification of older mass ligation techniques. To better understand and appreciate en masse or tourniquet lobectomy, one must review its inception and evolution. Historically, lobectomy is not a new procedure but has been intentionally performed since the late 1800 s. Probably the first surgeon to perform a planned pulmonary resection by mass ligation was Gluck $^{5}$ in 1881 in animals. Babcock ${ }^{6}$ in 1908 and Davies $^{7}$ in 1912 each did a lobectomy by anatomic dissection and ligation of individual hilar structures. Unfortunately, both patients died of overwhelming infection, which was the primary cause of death after these procedures. During the early 1900s, preoperative evaluation, postoperative care, and general anesthesia, as they are known today, were nonexistent. Trained anesthesiologists were unknown. With minimal preoperative preparation, absence of monitoring, and crude anesthesia, patients with advanced and usually bilateral bronchiectasis or tuberculosis underwent multistaged lobectomies that could take 3 to 4 months to accomplish. ., $^{8}$

In many of these cases, local intercostal anesthesia was used and patients were heavily sedated to suppress the cough reflex, which was thought to spread infection and, incidentally, disturbed the surgeon during the operative procedure. Patients had to breath spontaneously because positive-pressure ventilation was unfamiliar to most surgeons. Oxygen was not administered, only the pulse and blood pressure were monitored, endotracheal tubes were not used, antibiotics were unknown, and chest physiology, including paradoxic respiration, was poorly understood. Intrathoracic access was accomplished by resecting three to four ribs, and staged packing was required to develop adhesions to seal off selected areas of the thoracic cavity. When conditions seemed suitable, the fibrotic edematous hilum enveloped by engorged suppurative lymph nodes could be approached for resection. ${ }^{10,11}$

Although the procedure was called mass or tourniquet lobectomy, in reality there were numerous variations to this technique. Most commonly, a large tourniquet or a crushing clamp was applied to the hilum to secure hemostasis. Next the lobe was rapidly excised, usually without clamping the distal bronchus, so that pus exuded into the thorax. If possible, each hilar structure was then individually ligated, but usually only a running suture that included all structures could be achieved. The tourniquet or crushing clamp was then removed, and a second suture line of continuous or mattress sutures was placed in the large, previously clamped, devitalized area of tissue. Because contemporary principles of surgery had not yet evolved and, therefore, were not practiced, wound healing was severely jeopardized in these infected and devascularized tissues. One can only imagine the large amount of suppurative material that must have been massaged into the contralateral, dependent lung during these maneuvers. Because chest tubes were not routinely used, in most patients the wound was tightly packed with gauze. Obviously, intensive care units with cardioscopes, antibiotics, blood transfusions, arterial lines, 


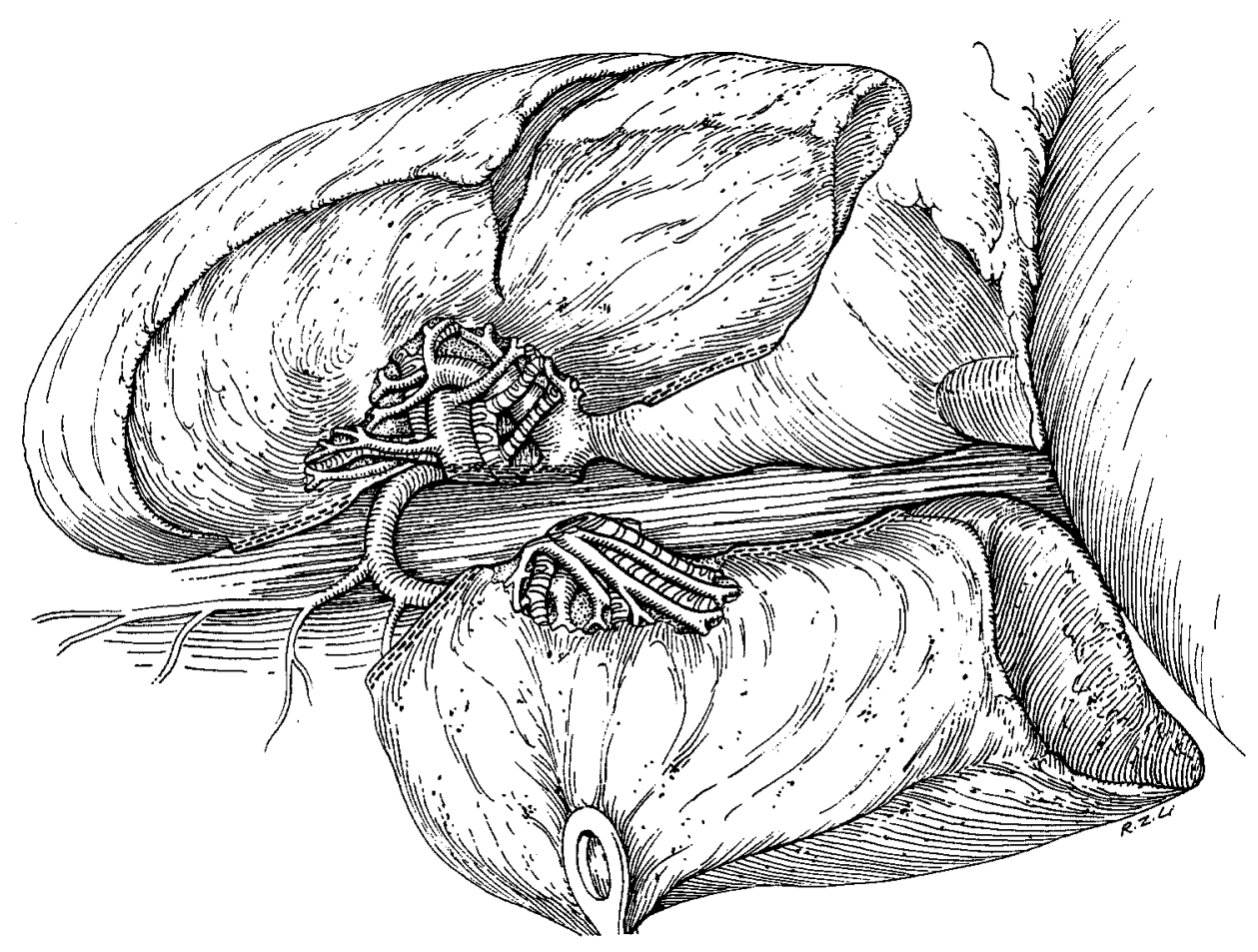

Fig. 5. Four rows of staples sealing all structures in their normal anatomic configuration.

ventilators, and portable chest x-ray machines were not available.

During that era, pioneers of lobectomy cautioned against hilar dissection and individual ligation for fear of spreading infection from opening inflamed tissue planes. ${ }^{12}$ It is fascinating, and even beyond comprehension, that this innovative, technically brilliant group of thoracic surgeons could salvage any patients with these detrimental and destructive procedures. A mortality of $50 \%$ was acceptable and a morbidity of $100 \%$ was always anticipated. Patients died of pulmonary and cardiac failure, hemorrhage, shock, and infection. In an era devoid of the routine, essential, supportive modalities currently available in any small community hospital, these imaginative surgeons were able to devise effective and serviceable operative techniques. Mass ligation or tourniquet lobectomy worked and was a major contributing factor enabling these pioneer surgeons to attain such enormous accomplishments.

The philosophy of mass ligation was logical and valid, but poor patient selection, ignorance of chest physiology and anesthesia techniques, absence of supportive modalities, and, most important, the underlying diseases of tuberculosis and bronchiectasis, without available antibiotics, were primarily responsible for the many failures. The infected edematous bronchi produced a high incidence of bronchopleural fistulas. In 1940, at the meeting of The American Association for Thoracic Surgery, Blades and Kent $^{13}$ rekindled interest in the isolation-ligation technique as a method for reducing the prevalence of bronchopleural fistulas. ${ }^{13}$ They presented a series of 10 patients. However, bronchial fistulas developed in six of them, for an incidence of $60 \%$. Although this technique did not seem to reduce the number of fistulas, its meticulous anatomic dissection became attractive and appealing to surgeons. Without any evidence or documentation of increased beneficial results, it was readily accepted as the standard operation for lobectomy. Concurrently, antibiotics were discovered and became very effective for the treatment of tuberculosis and bronchiectasis. The number of patients requiring surgical resections for these infections began to decrease, and as the prevalence of infection decreased, the prevalence of bronchopleural fistulas diminished. Those requiring the operation were now much better prepared and, consequently, became better operative risks. Another important factor was that the edematous, infected, fibrotic bronchi of tuberculosis and bronchiectasis were gradually being replaced by the then healthy, well-vascularized bronchus of patients with carcinoma. Other numer- 
ous and profound advances made during this period also improved patient survival. Most likely, the prevalence of bronchial fistula was decreasing because the underlying disease was changing from the infections of tuberculosis and bronchiectasis to the noninfected bronchus of carcinoma. Yet most surgeons attributed this major improvement primarily to the edifying, "clean" resections that could be accomplished by the isolation-ligation technique. They ignored the spectrum of profound advances and beneficial changes that were occurring in thoracic surgery at that time.

During the 1950s, Russian surgeons were beginning to develop and evaluate mechanical staplers. Even though these instruments were rudimentary and inefficient, they could be used for pulmonary resections. Numerous Russian surgeons performed lobectomies successfully by using these crude mechanical instruments to place a single staple line across the entire hilar area. ${ }^{14,15}$ Amosov and Berezovsky ${ }^{16}$ in 1960, in Istanbul, Turkey, described excellent results with a technique called single stage stapling. All structures at the root of the lobe were simultaneously stapled in their natural anatomic positions.

In the late 1960s and early 1970s, I had the opportunity to perform a large number of resections for patients with drug-resistant, advanced fibrotic calcific pulmonary tuberculosis. On occasion, because of extremely dense, adherent fibrosis, individual hilar structures could not be safely delineated, isolated, or ligated. A dense, calcific, and obliterated pericardium usually prevented intrapericardial dissection. Simultaneous ligation with mattress or running sutures, which included all of the vessels and the bronchus in one suture line, proved to be safe and expeditious. No bronchopleural fistulas developed, and each patient had a successful recovery. During the present era of sophisticated thoracic surgery, anatomic dissection with individual ligation of hilar structures in patients with an extensive hilar inflammatory reaction, even when performed by experienced surgeons at the Mayo Clinic, has resulted in a surgical mortality of $27.6 \%$ and a complication rate of $55.2 \% .{ }^{17}$ More than $17 \%$ of patients with benign disease died of uncontrollable hemorrhage during the operation. Because of poor results in the past with the isolation-ligation technique for patients with an infected bronchus, some contemporary surgeons advocate simultaneous ligation of all hilar structures as the procedure of choice when confronted with a dense, fibrotic, ligneous process. ${ }^{18}$
Gaskin and Bergmann ${ }^{19}$ in 1975 recommended simultaneous ligation of all pulmonary vessels after division of the bronchus. My experience with this technique has been favorable, without any adverse occurrences during the past 15 years, when used for lobectomy in the open chest. ${ }^{19}$

It must be remembered that resection of any tissue in the body usually compresses, distorts, and places arteries and veins in juxtaposition. Stapled gastric or colonic anastomoses contain systemic high-pressure arteries and veins in the most intimate of relationships, and yet fistulas are not being reported. Stapled wedge resections or even larger sublobar resections produce compression, distortion, and overlapping of pulmonary arteries, veins, and bronchi. Pulmonary parenchyma that envelopes and masks these structures obviously prevents direct visualization and awareness of these altered anatomic relationships. Despite millions of partial lung resections performed with staplers worldwide, reports alluding to arteriovenus or bronchovascular fistulas attributed to this technique are lacking.

Application of this same successful and proven technique to slightly larger, better exposed, and more easily visualized structures in the hilum is no different from performing a routine simple wedge resection. A carefully placed secure staple line maintaining all structures in their normal and natural anatomic configuration is easily accomplished with VATS. In fact, for VATS, it is safer than individual dissection and ligation, which can precipitate a hemorrhagic catastrophe. A second, distal staple line provides further assurance of complete ligation. This is not a blind, mass ligation that distorts, strangles, and crushes hilar structures; rather, it is a careful, meticulous application of the stapler, which individually but simultaneously ligates the pulmonary artery, pulmonary vein, and bronchus in their natural, anatomic configurations.

Simultaneously stapled lobectomy is feasible for patients with minimal pleural adhesions, well-developed fissures, and a pliable, elastic hilum free from enlarged lymph nodes. When there are obliterated fissures, enlarged matted invasive nodes, a fibrotic hilum, central or bulky lesions, calcific bronchi, chest wall invasion, or lesions crossing a fissure, simultaneously stapled lobectomy is to be avoided.

Currently, VATS techniques are being borrowed from procedures originally developed and intended for open thoracotomy. This is not only understandable but, historically, follows the path of natural evolution taken by most new techniques or innova- 
tions. Many of the open techniques that are being adapted for VATS lobectomy, however, can be cumbersome, awkward, and at times even dangerous. Not too many years ago, our predecessors cautioned against individual ligation for lobectomy because of the fear of spreading infection. ${ }^{9,10}$ They supported mass ligation as a safer and more effective technique, and it worked. Possibly, individual anatomic dissection and ligation of the hilum during VATS lobectomy should be avoided as being too dangerous because rapid access for hemorrhage might be unachievable. Instead, when VATS is being used, simultaneously stapled lobectomy should be advocated as a safer, more controllable procedure because it works. Interestingly, the full cycle for so-called mass ligation seems to be completed, but for different reasons in different categories of patients in whom markedly different techniques are used, and in different surgical eras. It was essential for resection when technology was completely lacking. Now, during an era of sophisticated, advanced technology, simultaneous hilar ligation once again seems to be a procedure with many advantages.

If VATS is to progress safely, expeditiously, and efficiently, then new techniques more innate, more specific, and even more natural for VATS must be developed.

\section{REFERENCES}

1. Lewis RJ, Caccavale RJ, Sisler GE, Mackenzie JW. One hundred consecutive patients undergoing videoassisted thoracic operations. Ann Thorac Surg 1992; 54:421-6.

2. Lewis RJ, Caccavale RJ, Sisler GE. Special report: videoendoscopic thoracic surgery. N J Med 1991;88: 473-5.

3. Mack MJ, Aronoff RS, Acuff TE, et al. Present role of thoracoscopy in the diagnosis and treatment of diseases of the chest. Ann Thorac Surg 1992;54:403-9.
4. Lewis RJ, Sisler GE, Caccavale RJ. Imaged thoracic lobectomy: Should it be done? Ann Thorac Surg 1992;54:80-3.

5. Gluck T. Experimenteller Beitrag zur Frage der Lungenextirpation. Berl Klin Wochenschr 1881;18:645.

6. Babcock WW. The operative treatment of pulmonary tuberculosis. JAMA 1908;50:1263-5.

7. Davies HM. Recent advances in the surgery of the lung and pleura. Br J Surg 1913;1:228-58.

8. Robinson S. The surgery of bronchiectasis. Surg Gynecol Obstet 1917;24:194-15.

9. Heuer G. The development of lobectomy and pneumonectomy in man. J Thorac CardiovasC Surg 1934;3: 560 .

10. Sarot I. Practical points in lobectomy. J THORAC Cardiovasc Surg 1939;8:300-8.

11. Eggers C. Lobectomy for carcinoma of the lung. J ThORAC CardiovasC SuRg 1934;4:211.

12. Lilienthal $H$. Extirpation of right lower lobe for septic bronchitis. Ann Surg 1915;61:103-5.

13. Blades B, Kent E. Individual ligation technique for lower lobe lobectomy. J Thorac Cardiovasc Surg 1940;10:84-101.

14. Rabinovich JJ. On the technique of combined resections of the bronchus and pulmonary artery with the use of autoplastic material. Grudnaya Khir 1967;6:50.

15. Berezovsky KK, Sabroda GS. On resection of lungs with the application of UKL-60. Novy Khirurgichesky Arkh 1959;1:18-24.

16. Amosov NM, Berezovsky KK. Pulmonary resection with mechanical suture. J THORAC CARDIOVASC SURG 1961;41:325-35.

17. McGovern EM, Trastek VF, Pairolero PC, Payne WS. Completion pneumonectomy: indications, complications and results. Ann Thorac Surg 1988;46:141-6.

18. Utley JR. Completion pneumonectomy and thoracoplasty for broncopleural fistula and fungal empyema. Ann Thorac Surg 1993;55:672-6.

19. Gaskin RJ, Bergmann M. Pneumonectomy by "en masse" stapling of hilar vessels. Ann Thorac Surg 1975;19:242-7. 